\section{Low-Refractive-Index Nanoporous Silica Improves Reflectivity of Dielectric Mirror}

For light-emitting diodes (LEDs) to become practical replacements for incandescent bulbs, the light must be extracted more efficiently than is currently possible. Since the active region emits light in all directions, the efficiency can be improved by adding a mirror to the back of the LED to redirect light out of the device. One such mirror is a triple-layer omnidirectional reflector (ODR), which uses a lowrefractive-index (low- $n)$, transparent dielectric in a semiconductor-dielectricmetal stack. As reported in the June 15 issue of Optics Letters (p. 1518), J.-Q. Xi and co-workers at Rensselaer Polytechnic Institute fabricated an ODR using nanoporous $\mathrm{SiO}_{2}$ as the low- $n$ dielectric.

The researchers used a spin-on sol-gel process to deposit a 105-nm-thick layer of nanoporous $\mathrm{SiO}_{2}$ (pore size, $\sim 4 \mathrm{~nm}$ ) on a 300- $\mu$ m-thick GaP wafer and then evaporated $500 \mathrm{~nm}$ of $\mathrm{Ag}$ onto the dielectric. They made two similar structures, one with an 89-nm-thick, dense $\mathrm{SiO}_{2}$ layer, and another without a dielectric layer. Ellipsometry revealed that the nanoporous $\mathrm{SiO}_{2}$ has a refractive index of only $n=1.23$, and the dense $\mathrm{SiO}_{2}$ has a refractive index of $n=1.457$.

Using a $632.8 \mathrm{~nm}$ coherent He-Ne laser, the group measured the angle-dependent reflectivity of the structures with the laser light incident from the $\mathrm{GaP}$-air side and used it to calculate the angle-integrated reflectivity $R_{\text {int }}$, a key figure of merit for an ODR. They coupled light into the device with a high-index fluid and prism, allowing for off-normal measurements up to $25.5^{\circ}$ for transverse electric (TE) polarization and $27.3^{\circ}$ for transverse magnetic (TM) polarization. Reflectivity measurements for the TE mode in the nanoporous $\mathrm{SiO}_{2}$ device yielded $R_{\text {int }}=99.9 \%$, while the $R_{\text {int }}$ of the dense $\mathrm{SiO}_{2}$ was $99.8 \%$, and the $R_{\text {int }}$ of the simple silver mirror was only $97.2 \%$. For TM polarization, which demonstrated more angular variation than the TE mode, the integrated reflectivities followed a similar trend, with $R_{\text {int }}$ values of $98.9 \%, 97.8 \%$, and $94.4 \%$ for the nanoporous $\mathrm{SiO}_{2} \mathrm{ODR}$, dense $\mathrm{SiO}_{2} \mathrm{ODR}$, and silver mirror, respectively.

The improved reflectivity of the nanoporous dielectric is consistent with its lower refractive index. The researchers said that such small increases in reflectivity translate into considerable reductions in mirror losses, especially when multiple reflections take place, thus suggesting that ODRs with nanoporous $\mathrm{SiO}_{2}$ could improve LED performance.

AMANDA GIERMANN

\section{D Defect Structures Embedded in Opal-Based Photonic Crystals}

Due to their potential to control light emission, routing, and filtering, photonic crystals can be employed as a tool to manipulate light for optical devices. To fabricate these materials, one route is to utilize the self-assembly of microspheres into colloidal crystals. In order to trap or localize light within these opal-based photonic crystals, researchers want to create controlled microscopic defects inside the lattice structure. As reported in the June 14 issue of Chemistry of Materials (p. 3069; DOI: 10.1021/CM050381L), Q. Yan, Z. Zhou, and X.S. Zhao from the National University of Singapore have engineered micrometer-scale defects consisting of polystyrene spheres within silicabased colloidal photonic crystals.

After fabricating the host opal by the assembly of monodisperse $0.39-\mu \mathrm{m}$ diameter silica colloidal microspheres using a vertical deposition method, the researchers deposited a thin layer of photoresist on top of the opal by spin-coating and then used conventional photolithography to create linear channels on the surface. Polystyrene microspheres were deposited in these channels either through spin-coating or by the vertical deposition method itself. The researchers then dissolved the photoresist and re-grew the host silica colloidal crystal by vertical deposition. By combining standard lithography with self-assembly, the researchers could embed polystyrene microspheres within the silica colloidal crystal. Another key advantage of this simple process, according to the researchers, is that the thickness of the defect layer (photoresist) can be controlled by merely changing the spinning speed and deposition time.

Besides straight linear channels, other defect structure geometries such as S-bend, Y-branch, X-cross, and rings have been fabricated on the colloidal crystal surface. These configurations are useful for waveguiding and filtering applications. Zhao said that this technique is inexpensive and straightforward to implement, and may be useful for non-photonic devices, such as filtering elements in microfluidic channels. TUSHAR PRASAD

\section{Molecular-Scale Si Nanowires Enable Coherent Single-Charge Transport}

Nanoscale electronics require building blocks that have controllable properties and can easily be arranged into complex, integrated structures. The "bottom-up" approach to creating such structures is advantageous because critical feature sizes are defined during synthesis, allowing for atomic-scale control over size and properties. Although isolated carbon nanotubes exhibit exceptional properties, silicon nanowires show promise for large-scale integration due to the ability to reproducibly control their size and properties. However, fundamental electrical transport properties have not been reported for small-diameter Si nanowires-that is, strong confinement behavior in one dimension. In the May 6 issue of NanoLetters (p. 1143; DOI: 10.1021/nl050783s), Z. Zhong, Y. Fang, W. Lu, and C.M. Lieber of Harvard University have now characterized the electrical transport of chemically synthesized, molecular-scale Si nanowires 3-6 nm in diameter.

The study probed the low-temperature $(4.2 \mathrm{~K})$ transport properties of such Si nanowires assembled into single-electron transistors (SETs). The SETs were formed from single-crystal Si nanowires on 50-nmthick thermal oxide on degenerately doped Si substrates with 50-nm-thick Ni sourcedrain contacts defined by electron-beam lithography. Electrical measurements on single Si nanowires demonstrated regular Coulomb blockade oscillations consistent with phenomena resulting from singlecharge tunneling through a single quantum structure, not a series of interconnected quantum dots. Coherent charge transport was further demonstrated through discrete single-particle quantum levels extending across the SET devices over length scales of up to $200 \mathrm{~nm}$, in marked contrast to predictions for the properties of lithographically patterned planar silicon. The researchers said that this molecularscale Si nanowire system can serve as a potential building block for both conventional and quantum electronics. The researchers also said that the richness of the transport phenomena observed in this initial study suggested that correlation effects beyond the constant interaction model (in which each electron is assumed to be independent) may be important in describing the behavior of these SETs in the few-charge regime, where electron behavior is likely quantized.

ADITI RISBUD

\section{Researchers Elucidate the Structure of the Protein-Mineral Interface in Bone}

Bone is a material with remarkable mechanical properties, which have been the focus of numerous investigations. Although bone is relatively lightweight, it is strong, robust, and tough. The reasons for these unusual properties are not yet well understood. In contrast to many synthetic materials, natural bone features a composite-like nanostructure comprising 
an organic matrix dominated by collagen, with nanoscale inorganic crystals composed mostly of hydroxyapatite. These two phases are arranged in a staggered nanostructure of largeaspect-ratio inorganic crystals embedded in the organic matrix. In the June 14 issue of Chemistry of Materials (p. 3059; DOI: $10.1021 / \mathrm{cm} 050492 \mathrm{k}), \mathrm{C}$. Jaeger of the Federal Institute of Materials Research and Testing in Berlin, Germany; N.S. Groom of the University of Cambridge; E.A. Bowe of the Royal Veterinary College in London; R.C. Murray of the Animal Health Trust in Suffolk; and their colleagues have reported solid-state nuclear magnetic resonance (NMR) studies of the interface of the organic phase and inorganic crystallites in bone.

The researchers believe that understanding these organic-inorganic interface properties is the key to understanding the macroscopic material properties of bone.

"Not only would such knowledge help us understand the superior properties of bone, but it may also assist in the design of new, synthetic bone-like and other materials," said coresearcher M.J. Duer of the Department of Chemistry at the University of Cambridge.

Based on a series of NMR experiments, the scientists proposed a quantitative structural model of the possible binding site for a carboxylate group at the surface of the calcium phosphate phase in bone. The results demonstrated that the glutamate binding proteins interact very strongly with $\mathrm{Ca}^{2+}$ ions on the surface of the mineral phase.

"As collagen itself contains very little glutamate or glutamine, the proteins associated with binding to the mineral phase must be in the non-collageneous part of the protein matrix," said Duer. These results suggest that the collagen itself does not bind to the mineral particles, but that an additional protein interface layer is responsible for the binding.

"Since the $\mathrm{P}-\mathrm{OH}$ groups of the inorganic platelets are sufficiently close to the carboxylate or carbonyl groups of the organic material, hydrogen bonds may play a critical role in determining the interface properties," said Duer. The researchers report that this hypothesis is further supported by rotational echo double resonance (REDOR) experiments.

MARKUS J. BUEHLER

\section{"Top-Down" Technique Allows for PRINTing of Shape-Specific Nanobiomaterials}

In recent years, biomaterials have played a pivotal role in the success of drug delivery and medical devices. To achieve optimal performance in drug, targeting, and recognition applications, these materials should exhibit programmed responses to external stimuli and recognition events. Examples of such materials are socalled "smart" particles, whose form (i.e., morphology and composition) dictates function. In the June 2 issue of the Journal of the American Chemical Society (DOI: 10.1021/ja05/977c), J.P. Rolland, B.W. Maynor, L.E. Euliss, A.E. Exner, G.M. Denison, and J.M. DeSimone of the University of North Carolina at Chapel Hill outline a method for fabricating arrays of these particles using a "top-down" approach called PRINT (particle replication in nonwetting templates). Unlike traditional imprint lithography techniques, PRINT uses a nonwetting layer of fluorinated surfaces to confine a liquid precursor inside the features of a photocurable perfluoropolyether (PFPE) mold, allowing for the production of isolated particles. The researchers said that the technique enables the fabrication of repeatable features at dimensions of $100 \mathrm{~nm}$ with control over particle size, shape, and composition.

The researchers started with master templates that were either obtained commercially or fabricated in silicon using electronbeam lithography. PFPE replica molds were then made by photochemically curing a dimethacrylate-functionalized PFPE oligomer

\section{See More... \\ Do More...}

\section{Integrated Solutions for Integrated Applications}

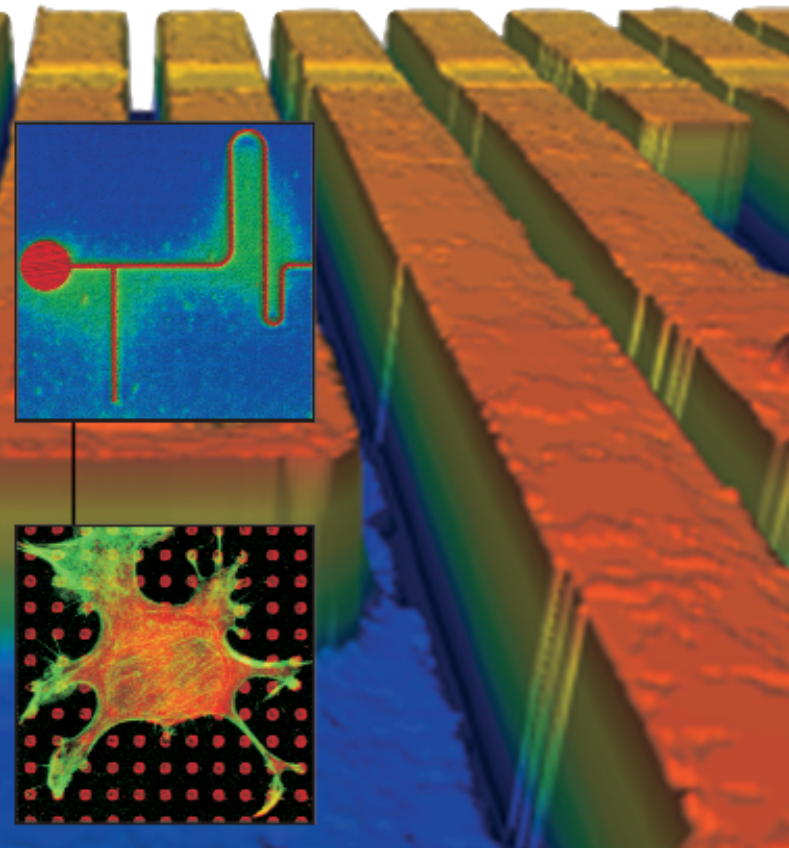

\section{Carl Zeiss LSM 5 PASCAL}

Confocal Laser Scanning Microscope

Insight into Biology and Materials Science through:

- 3D Imaging

- Surface topography

- Volumetric measurement

- Multi-label Imaging

- Fluorescence \& Reflected light imaging

Applications in Research and Quality Assurance:

- Microfluidics

- Microarrays

- Microreplication

- Sensors

- Lab-on-Chip

Carl Zeiss Microlmaging Inc.

Thornwood, NY 10594

800.233 .2343

micro@zeiss.com

zeiss.com/lsm-mat

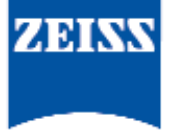

We make it visible. 